\title{
GAG-Ersatztherapie bei chronischen Zystitiden: Studienanalyse bestätigt Chondroitinsulfat
}

- Chronische Zystitiden wie die Interstitielle Zystitis, die überaktive Blase, chronisch rezidivierende Harnwegsinfekte oder die radiogene Zystitis haben häufig eine gemeinsame Ursache: Die äußerste Blasenschutzschicht - die Glykosaminoglykan (GAG)Schicht ist defekt. Reizende Substanzen können Rezeptoren der Blasenwand irritieren, die Patienten leiden unter starkem Harndrang, erhöhter Miktionsfrequenz oder Schmerzen im Beckenbereich. Eine kausalnahe und nachhaltige Behandlung chronischer Zystitiden verspricht die GAG-Ersatztherapie. Dafür stehen Substanzen wie Chondroitinsulfat, Heparin, Hyaluronsäure oder Pentosanpolysulfat zur Verfügung. Welche
Substanz vor dem Hintergrund kontrollierter, randomisierter klinischer Studien die besten klinischen Effekte zeigt, untersuchten Prof. Helmut Madersbacher, Wien, et al. auf Basis von 27 klinischen Studien [Madersbacher et al., Neurourology and Urodyanmics; DOI 10.10002/nau].

Aus dem Review geht hervor, dass lediglich für Chondroitinsulfat, eine Kombination aus Chondroitinsulfat und Hyaluronsäure sowie Pentosanpolysulfat publizierte randomisierte Studien vorliegen. Zwei Studien, in denen die Wirkung von Hyaluronsäure gegenüber Placebo untersucht wurde, wurden aufgrund fehlender signifikanter Ergebnisse nicht veröffentlicht. Insgesamt wurden im Rahmen von dokumentierten, publizierten Studien am meisten Patienten mit einer Chondroitinsulfat-GAG-Ersatztherapie behandelt. Fazit der Autoren: Auf Basis der Studiendaten ist Chondroitinsulfat in einer Konzentration von $0,2 \%$ (Gepan $^{\circledast}$ instill) für die intravesikale GAG-Ersatztherapie zu favorisieren. Es gibt keine Hinweise für eine überlegene Wirkung der 2,0\%igen Chondroitinsulfatlösung gegenüber $0,2 \%$ igen Chondroitinsulfat. Zudem zeigte eine Studie mit 2,0\% Chondroitinsulfat keine statistisch signifikanten Ergebnisse. Gepan ${ }^{\circledast}$ instill versorgt die GAG-Schicht mit ihrem Baustein Chondroitinsulfat und behebt den Defekt der Blasenschutzschicht. Davon können Patienten aller Formen der chronischen Zystitis profitieren.

Nach Informationen von

Pohl-Boskamp, Hohenlockstedt

\section{Auszeichnung für innovativstes Produkt 2012 beim Urologen}

— Zytiga ${ }^{\oplus}$ (Abirateronacetat) ist in diesem Jahr mit dem Preis „Innovativstes Produkt" beim Urologen ausgezeichnet worden. Die Umfrage erfolgte im Rahmen des Pharma Trends, bei dem seit dem Jahr 2000 jährlich im Auftrag der Zeitschrift PharmaBarometer Mediziner aus verschiedenen Facharztgruppen online und telefonisch in standardisierten Fragebogen gebeten werden, ihre Meinung über Unternehmen der pharmazeutischen Industrie und deren Produkte abzugeben. Die Frage, welches Medikament für die Ärzte das innovativste Produkt des Jahres ist, wird offen ohne jegliche Vorgaben gestellt. Die einfache Stimmenmehrheit entscheidet über den Gewinner. 2012 wurden 1.500 Mediziner aus zehn Facharztgruppen befragt, unter ihnen 150 Urologen.

Abirateronacetat ist ein steroidaler Androgen-Biosynthese-Inhibitor und wurde 2011 in Kombination mit Prednison oder Prednisolon zur Behandlung des metastasierten kastrationsresistenten Prostatakarzinoms ( $\mathrm{mCRPC}$ ) bei erwachsenen Männern zugelassen, deren Erkrankung während oder nach einer Docetaxel-haltigen Chemotherapie progredient ist. Die Zulassung basiert auf der Phase-III-Studie COU-AA-301. In dieser Studie hatten 1.195 Patienten mit mCRPC nach Versagen von ein bis zwei Chemotherapie-Regimen, von denen zumindest eines Docetaxel-haltig war, Abirateronacetat oder Placebo - in Kombination mit Prednison/Prednisolon - erhalten [de Bono JS et al. N Engl J Med 2011; 364: 1995-2005]. Abirateron verlängerte gegenüber Placebo das Gesamtüberleben signifikant um 4,6 Monate (median 15,8 vs. 11,2 Monate, $\mathrm{HR}=0,74, \mathrm{p}<0,0001$ ) [Fizazi K et al. ECCO 2011: Abstract 7000 (oral presentation)].

\section{PCa: Wissen mit Spendenscheck für Kinderhospiz belohnt}

- Unter dem Motto „Wissen testen und Gutes tun“ waren die Besucher des diesjährigen Kongresses der Deutschen Gesellschaft für Urologie aufgerufen, an einem interaktiven Wissens-Quiz rund um die Therapie des Prostatakarzinoms (PCa) teilzunehmen. Richtig beantwortete Fragen wurden mit „barer Münze“ aufgewogen.

Über 200 Teilnehmer folgten dem Aufruf von Orion Pharma und der Pharma-Marketingagentur Juwi MacMillan und beteiligten sich an dem Spendenquiz. Sie stellten sich den Fragen rund um die Therapie des Prostatakarzinoms mit Fokus auf dem Histrelin-Jahresimplantat (Van$\operatorname{tas}^{\circledast}$ ). Das Histrelin-Jahresimplantat ist seit 2008 zur antihormonellen PCa-Therapie bei Männern mit fortgeschrittenem Prostatakarzinom zugelassen. Es gibt täglich $50 \mu \mathrm{g}$ Histrelinacetat ab und senkt bereits innerhalb von vier Wochen die Testosteronwerte unter das mit $20 \mathrm{ng} /$ dl definierte Kastrationsniveau. Die Testosteronwerte steigen weder nach dem Wechsel des Implantats noch nach vier Behandlungsjahren. Jede im Quiz richtig beantwortete Frage hatte einen Wert von 5 EUR, der Spendenzähler stoppte bei 3.035 EUR. Dieser Betrag wurde auf 3.500 EUR aufgerundet. Diese Summe ging an den Verein Kinderhospiz Bärenherz e.V. Die Spende helfe dabei, dem einzigen stationären Kinderhospiz in Sachsen und Sachsen-Anhalt auch zukünftig zuverlässiges Personal zur Verfügung zu stellen, so Hubertus Freiherr von Erffa, Vorsitzender des Vereins.

Ute Ayazpoor

Spendenscheckübergabe im Rahmen des 64. Kongresses der Deutschen Gesellschaft für Urologie, Leipzig, 28. September 2012, Veranstalter: Orion Pharma, Hamburg 